Hobson, P. N., MANN, S. O. \& OxFond, A. E. (1958). J. gen. Microbiol. 19, 462-472

\title{
Some Studies on the Occurrence and Properties of a Large Gram-Negative Coccus from the Rumen
}

\author{
By P. N. HOBSON, S. O. MANN AND A. E. OXFORD* \\ Rowett Research Institute, Bucksburn, Aberdeenshire
}

\begin{abstract}
SUMMARY: A large Gram-negative coccus was isolated in a lactate-containing medium from the rumen of young calves. This organism is anaerobic and ferments only lactate, glucose, fructose, maltose, sucrose, mannitol and sorbitol. The coccus is found in large numbers $\left(c .10^{9} / \mathrm{ml}\right.$.) in the rumen contents of young calves, but is present only in small numbers or is absent from the rumens of older cattle and sheep. An attempt made to establish the organism in large numbers in a sheep rumen was unsuccessful. The possibility of the coccus being a normal inhabitant of the throat of adult animals was also examined. Five serological types were obtained. The distribution of these types in the same animal over a period, and in different animals, appears to be random. The distribution of a small lactate-fermenting coccus (Veillonella gazogenes) is also described.
\end{abstract}

During the last few years a large number of bacteria have been isolated from the rumens of cattle and sheep, and some of these have been the subjects of extensive study. The present paper deals with the properties and occurrence of a large Gram-negative coccus which has been found to be common in the rumens of young animals, but less so in older animals. The organism was first isolated by chance in media used for cultivating lactobacilli and when its properties became known it was sought specifically in rumen contents. A preliminary communication about this organism has already been made (Hobson, Mann \& Oxford, 1958).

\section{METHODS}

Media. Tomato extract agar was prepared by the method of Rogosa et al. (1953). The semi-solid medium contained Bacto casitone, 6 g.; Bacto yeast extract, 2 g.; L-cystine, $0 \cdot 3 \mathrm{~g}$.; thioglycollic (mercaptoacetic) acid, $0 \cdot 12 \mathrm{ml}$; $\mathrm{NaCl}, 2$ g.; agar, $0 \cdot 3 \mathrm{~g}$.; sodium lactate solution $(70 \%$, w/w $), 5 \cdot 7 \mathrm{ml}$; resazurin, $0.4 \mathrm{ml}$. of $0.1 \%(\mathrm{w} / \mathrm{v})$ solution; distilled water, $400 \mathrm{ml}$. Sterilized by autoclaving at $121^{\circ}$ for $15 \mathrm{~min}$. The $\mathrm{pH}$ value of the medium was 6.9-7.0. Increasing the agar concentration to $2 \%$ gave solid thioglycollate medium. Johns's medium was prepared as described by Johns (1951). This was made into Chocolate Johns's Agar (CJA) medium by addition of $5 \%(\mathrm{v} / \mathrm{v})$ heated blood. Elsden's media A and B were prepared by the method of Elsden et al. (1956). The semi-solid medium ( $9 \mathrm{ml}$.) was incubated in tubes $\left(6 \mathrm{in} . \times \frac{5}{8} \mathrm{in}\right.$.) in air, other media under $5 \%(\mathrm{v} / \mathrm{v}) \mathrm{CO}_{2}+95 \%(\mathrm{v} / \mathrm{v}) \mathrm{H}_{2}$, and all at $38^{\circ}$. Sodium lactate solution was a $70 \%(\mathrm{w} / \mathrm{w})$ solution (Hopkin and Williams Ltd.,

* Present address: 'Pukenui', Northland, New Zealand. 
Chadwell Heath) and the sugars used were the standard grades supplied by L. Light, Colnbrookdale, T. Kerfoot, Vale of Bardsley, or British Drug Houses Ltd. All media mentioned later in the text contained sodium lactate and no carbohydrate unless described otherwise.

Isolation of the organism. The final isolation method was as follows. Samples were taken from animals fitted with permanent rumen cannulae or by stomach tube, and when necessary lightly centrifuged to remove the larger debris. Serial tenfold dilutions were then made in the semi-solid medium and these were incubated for 2 days. The tubes were examined for growth of the large coccus and subcultures were made, generally from the highest dilutions in which growth was occurring, on to CJA medium or on solid thioglycollate medium. Further subcultures were made into Robertson's cooked meat medium and back on to CJA or solid thioglycollate media until a pure culture was obtained.

Preservation of the organism. Stock cultures were kept in Robertson's cooked meat media subcultured every 5-7 days. Representative cultures of the different serological types, grown in Johns's medium, were freeze dried from glucose + serum suspension after the organisms had been washed once.

Biochemical reactions. Fermentation reactions were determined by using the semi-solid medium adjusted to $\mathrm{pH} 7 \cdot 0-7 \cdot 2$, containing $1 \%(\mathrm{w} / \mathrm{v})$ substrate and $0 \cdot 1 \%(w / w)$ of a $1 \cdot 6 \%(w / v)$ ethanolic solution of bromocresol purple. Tests for formation of $\mathrm{H}_{2} \mathrm{~S}$ and indole were made with the semi-solid medium, the oxidase and catalase tests on CJA medium, and gelatin liquefaction in the semi-solid medium with the agar replaced by $12 \%(\mathrm{w} / \mathrm{v})$ gelatin.

Determination of fermentation products. Total volatile fatty acids were determined by steam distillation in the apparatus of Markham (1942) and titration of the distillate with $0.05 \mathrm{~N}-\mathrm{NaOH}$ in a stream of $\mathrm{CO}_{2}$-free air. For qualitative determination of the acids present the distillate was evaporated to a suitable volume and analysed chromatographically on filter paper by the method of Elsden \& Lewis (1953). The acids were analysed quantitatively, after a second distillation with mercuric salts (Friedemann, 1938) by evaporating the alkaline extract to dryness, and extracting with a chloroform + butanol mixture (Elsden, 1946). A portion of this solution was titrated with ethanolic $\mathrm{NaOH}$ to determine the total acid present, and further samples were analysed on silica columns as described by Moyle, Baldwin \& Scarisbrick (1948). Gases were determined by cultivating the organism in a completely filled flask connected by a capillary to a mercury filled gas burette. $\mathrm{CO}_{2}$ in the gas evolved was absorbed in barium hydroxide solution and the residual gas, which was explosive, was assumed to be hydrogen.

Serological methods. Preparations of antigens were made from organisms from a $48 \mathrm{hr}$. culture in Johns's medium; they were centrifuged down, washed three times with saline, suspended in saline, and heated at $60^{\circ}$ for $1 \mathrm{hr}$. These suspensions, with the addition of $0.5 \%(\mathrm{w} / \mathrm{v})$ phenol, were kept at $2^{\circ}$.

The method given by Browning \& Mackie (1949) for preparation of antisera to meningococci was used for preparing antisera. Usually two series of injections produced a good antiserum. Doubling dilutions of the antisera were 
tested for agglutination reactions in tubes. Rapid serological tests were made with undiluted antisera for slide reactions. Antisera which cross-reacted in agglutination tests were absorbed by a heavy suspension of the appropriate organism until no further reaction occurred.

Fluorescent antisera were prepared and liver-absorbed as described by Hobson \& Mann (1957). Tests were made by methods also described in this paper.

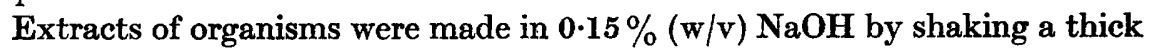
suspension with ballotini beads for $45 \mathrm{~min}$. at room temperature. The disintegrated cells were removed by filtration through a Berkefeld filter and the extract neutralized with dilute $\mathrm{HCl}$ when necessary.

Precipitin tests were carried out by layering the extract on to the antiserum in a small tube; an interfacial precipitate was looked for after incubation at $37^{\circ}$ for $30 \mathrm{~min}$.

Growth at different $p H$ values. This was tested by using the semi-solid medium buffered with citric acid $+\mathrm{KH}_{2} \mathrm{PO}_{4}$ mixtures. The $\mathrm{pH}$ values of the cultures and uninoculated controls incubated together were measured with a Pye pH meter.

Tests for formation of iodine-staining polysaccharide. The colonies growing on CJA medium containing either lactate, glucose or maltose were flooded with iodine at intervals, and the reactions of colonies and individual organisms noted.

Identification of the small Gram-negative coccus sometimes cultured with the large coccus. The fermentation reactions of the small coccus were determined by using a semi-solid medium; other tests were as for the large coccus. The small and large cocci could be distinguished on CJA medium, the large coccus having a greenish colony, and the small coccus a greyish-white colony.

\section{RESULTS}

The first isolations were made on tomato extract agar which gave growth corresponding to $10^{4}$ organisms $/ \mathrm{ml}$. rumen contents from a 5-day-old calf. When it was found that the organism metabolized lactate, isolation in a lactatecontaining medium was attempted and it was found that the procedure described above was satisfactory. In a later experiment samples from calves 1 and $m$ at 3 weeks old (Table 4) were diluted and cultured in Elsden's media A and $\mathbf{B}$. In medium $\mathbf{A}$, containing starch, the coccus grew only in dilutions corresponding to numbers of $100 / \mathrm{ml}$. rumen contents, and small Gram-positive cocci, probably Streptococcus bovis, grew in dilutions corresponding to $10^{9} / \mathrm{ml}$. In medium $\mathrm{B}$, containing lactate, the large coccus grew in dilutions corresponding to $10^{9}$ or $10^{10} / \mathrm{ml}$. which compared well with the results of the normal isolation procedure. Elsden et al. (1956) found that a lactate-containing medium inoculated with rumen contents produced a dense growth of Veillonella gazogenes which completely overgrew the large coccus and made it impossible to isolate. Although we generally obtained a mixed growth of organisms in the lactate-containing medium, only in one or two cases were small Gram- 
negative cocci present in numbers great enough to prevent the isolation of the large coccus. A number of isolations were made of small cocci which from their morphology, ability to ferment only lactate, pyruvate, malate and fumarate to give acid and gas, together with the fact that they were anaerobic, were identified as Veillonella gazogenes (Johns, 1951).

The large coccal organism is usually Gram-negative, but some organisms have a more purplish colour than the majority and occasionally a coccus is found which seems to be Gram-positive. The morphology of the coccus is shown in Pl. 1, fig. 1-4. The diameter of cocci in fixed preparations is usually about 1.5 $\mu$, but this varies and occasional large forms are found. These large forms, which often become granular, become more numerous as the cultures become older until they predominate. Old cultures autolyse rapidly with the formation of granular masses of debris, and the autolysis seems to be most rapid between pH 5 and 7. The cocci usually occur in pairs, and sometimes singly, but chains are rare, except in young cultures. It has not been found possible to distinguish a capsule by normal negative staining methods, but fluorescent antisera seem to demonstrate a small capsule or slime layer varying in thickness from coccus to coccus. The organism generally occurs in the rumen as isolated diplococci and in small groups, but one large group reacting with fluorescent antiserum is shown in Pl. 1, fig. 5, 6.

There are some differences in colony type amongst the organisms isolated. Serological type 2 has colonies $1-1.5 \mathrm{~mm}$. diameter, with raised centres, greenish yellow in colour, and with flat greyish peripheries with an uneven edge and smooth surface. Smaller colonies do not show the flat periphery. The other types have a similar colony, but the centre is not as raised or pigmented and the flat periphery is more extended. Deposits of organisms from liquid cultures also have a greenish colour.

The organism is an obligate anaerobe and in liquid or on solid media is grown under $5 \%(\mathrm{v} / \mathrm{v}) \mathrm{CO}_{2}+95 \%(\mathrm{v} / \mathrm{v}) \mathrm{H}_{2}$, but it does not need this concentration of $\mathrm{CO}_{2}$ and will grow under $\mathrm{H}_{2}$ alone or in the depths of the semi-solid medium. In liquid cultures it needs a large inoculum to start growth, but it does not require a rumen liquid supplement as do some rumen bacteria.

The fermentation reactions are shown in Table 1 . Twenty-one isolations, representing serological types $1-\mathbf{3}$, were tested for fermentation reactions. With the exception of sorbitol, which was fermented by types 1 and 3 , but not by type 2 , no variation in reactions was found. $\mathrm{H}_{2} \mathrm{~S}$ is formed, but not indole, catalase or oxidase. Gelatin is not liquefied.

When grown on lactate the $\mathrm{pH}$ value initially decreases, but then increases to 7 or slightly above; $\mathrm{CO}_{2}$ and $\mathrm{H}_{2}$ and volatile fatty acids are formed. In one experiment the acids formed (as m-equiv $\%$ of total m-equiv of acid formed) were: valeric $12 \cdot 6$, butyric $13 \cdot 7$, propionic $38 \cdot 2$, acetic $35 \cdot 5$. In the same basal medium growth on glucose was slower, the final $\mathrm{pH}$ value being $4.9 ; \mathrm{CO}_{2}$ and $\mathrm{H}_{2}$ were again formed together with fatty acids. In this case little or no propionic acid, but some caproic acid, was formed. The proportions of the different acids differed in different experiments, and at different times in the same experiment, but the same acids were found each time. In one experiment 


\section{Table 1. Fermentation reactions of the large Gram-negative coccus}

Semi-solid medium as described in text. Substrates $1 \%(w / v)$. Incubation 14 days at $37^{\circ}$.

\begin{tabular}{lclc} 
Substrate & Reaction & \multicolumn{1}{c}{ Substrate } & Reaction \\
Fructose & + & Aesculin & - \\
Glucose & + & Salicin & - \\
Galactose & - & Mannitol & + \\
Xylose & - & Sorbitol & + \\
Arabinose & - & Dulcitol & - \\
Rhamnose & - & Inositol & - \\
Maltose & + & Glycerol & - \\
Sucrose & + & Adonitol & - \\
Trehalose & - & Sod. lactate & + \\
Lactose & - & Sod. succinate & - \\
Raffinose & - & Sod. fumarate & - \\
Dextrin & - & Sod. formate & - \\
Starch & - & Sod. malate & - \\
Inulin & - & Sod. alanate & -
\end{tabular}

+ , Acid plus gas; -, no action. Growth on sucrose was slower than on other sugars; fermentation of sorbitol varied with the serological type (see text).

the acids formed were: caproic $5.9 \%$, valeric $6.7 \%$, butyric $52 \cdot 1 \%$, propionic $1.3 \%$, acetic $33.9 \%$, respectively, of the total volatile fatty acids recovered after distillation with mercuric salts. In cultures grown on glucose there was always a volatile acid present (about 6-10\% of the total) which was destroyed during this distillation and which was probably formic acid. The only carbohydrate to be detected chromatographically in the culture filtrate after growth on glucose was glucose; no soluble oligosaccharides appeared to be formed.

Growth occurred over the range of $\mathrm{pH} 4 \cdot 8-8 \cdot 1$. The preliminary tests for formation of iodine-staining polysaccharide in the cocci showed that they became filled with material giving a dark brown, often granular, glycogen-like stain with iodine after about $40 \mathrm{hr}$., and that this was lacking after $24 \mathrm{hr}$. incubation; but these times varied with the rate of growth. The colonies became dark brown staining with iodine at the same time, and this could easily be distinguished from the normal light brown colour of the colonies with iodine.

The results of experiments in which counts of the numbers of cocci present in the rumen contents of a number of calves and heifers were made are shown in Table 2. The fluorescent antiserum technique was used to test for the presence of cocci in these and other rumen contents as an additional check on the isolations and also to try to determine whether the large cocci visible in some samples, but which would apparently not grow on the media used, were in fact the lactate-utilizing organism. The calves were getting a variety of diets at the times of sampling, from a completely milk diet to a concentrate and hay diet for the oldest ones. The environments of the animals also differed, and the samples were taken at various times over some two years. Table 3 shows the distribution of these cocci in four adult sheep sampled over a period. During this time the environments and diets were constant. Sheep 21 was getting a diet of hay, potato starch and concentrates, sheep 86 and 879 concentrates and hay, and sheep 9 hay and grass cubes. Two calves, 1 and $\mathrm{m}$, 
Table 2. Distribution of large Gram-negative cocci in cattle

\begin{tabular}{|c|c|c|c|}
\hline Animal & $\begin{array}{c}\text { Age at } \\
\text { time of } \\
\text { sampling }\end{array}$ & $\begin{array}{c}\text { Isolation } \\
\text { at } / \mathrm{ml} \text {. }\end{array}$ & $\begin{array}{c}\text { Found with } \\
\text { fluorescent antisera } \\
\text { types } 1 \text { and } 2\end{array}$ \\
\hline \multicolumn{4}{|l|}{ Calf } \\
\hline $\mathbf{a}$ & 20 weeks & $10^{1}, 10^{2}$ & - \\
\hline b & 20 weeks & 0 & - \\
\hline c & 8 weeks & $10^{9}$ & . \\
\hline d & 6 weeks & $10^{10}$ & ++ \\
\hline $\mathbf{e}$ & 8 weeks & $10^{9}$ & . \\
\hline $\mathbf{f}$ & 5 weeks & $10^{4}$ & - \\
\hline $\mathbf{g}$ & 7 weeks & $10^{10}$ & $t+$ \\
\hline h & 7 weeks & $10^{8}$ & . \\
\hline $\mathbf{i}$ & 9 weeks & $10^{8}$ & . \\
\hline j & 3 weeks & $10^{10}$ & $\pm *$ \\
\hline $\mathbf{k}$ & 6 weeks & - & $+t$ \\
\hline \multicolumn{4}{|l|}{ Heifers $\dagger$} \\
\hline 6 & 15 months & 0 & \pm or - \\
\hline \multicolumn{4}{|l|}{ Heifer } \\
\hline $\begin{array}{l}1 \\
\mathbf{2} \\
\mathbf{3}\end{array}$ & 15 months & $\begin{array}{c}0 \\
0 \text { or } 10^{5} \S \\
0\end{array}$ & $\begin{array}{c}+ \\
\pm \text { or }- \text { or } \\
++\end{array}$ \\
\hline \multicolumn{4}{|l|}{ Heifer } \\
\hline $\begin{array}{l}1 a \\
2 a \| \\
3 a\end{array}$ & 15 months & $\begin{array}{c}0 \\
0 \text { or } 10^{5} \xi \\
0\end{array}$ & $\begin{array}{c}- \\
- \text { or } \pm 8 \\
\pm\end{array}$ \\
\hline
\end{tabular}

Calves of the same age were not necessarily sampled at the same times. The tests cover a period of some 2 years.

* Isolated cocci were different serological type to antisera used for testing.

$\dagger$ Grass diet.

$\mp$ Silage diet. Samples at intervals over 1 month.

$\$$ Corresponding samples.

II Roots diet.

$\rightarrow$, No large cocci reacting; \pm , few;,+++ , increasing numbers.

., No test.

were sampled for these cocci at intervals from 3 weeks old. The diets of both were the same, the calves being weaned 1 to 2 days before the first sample and thereafter a concentrate mixture, made up from groundnut and molasses meals, flaked maize, bruised oats, minerals and vitamins, and a little hay were given. The cocci were present in both calves for the whole time of sampling (Table 4). The isolation of these cocci from kids was not attempted but observations of stained films of kid rumen contents showed very few large Gramnegative cocci.

\section{Serology}

An antiserum to one of the original isolates from a calf was prepared and other isolates were tested against this antiserum. One of the isolates did not react with this antiserum in high dilution and this was used for preparing a second antiserum. Subsequent isolates of large cocci on plate cultures, usually derived from the highest dilution primary cultures which showed growth, were rapidly tested by the slide-agglutination technique. Only the representatives of new types were then kept for more extensive tests. Tests of some 250 isolates 
Table 3. Distribution of large Gram-negative cocci in sheep

Found with

Isolation fluorescent antisera

Sheep Test no. at/ml. types 1 and 2

\begin{tabular}{|c|c|c|c|}
\hline No. 21 & 1 & & \pm \\
\hline & $\begin{array}{c}2,3,4 \\
5\end{array}$ & 0 & - \\
\hline & 6 & $10^{4}$ & . \\
\hline & 7 & $10^{5}$ & . \\
\hline & 8 & $10^{4}$ & . \\
\hline No. 879 & 1 & & - \\
\hline & 2 & $10^{1}$ & • \\
\hline & 3 & $10^{4}$ & . \\
\hline & 4 & $10^{6}$ & . \\
\hline No. 9 & 1 & $\mathbf{0}$ & \pm \\
\hline & 2 & 0 & \\
\hline & $\mathbf{3}$ & 0 & . \\
\hline & 4 & 0 & - \\
\hline No. 86 & 1 & o & \\
\hline
\end{tabular}

Symbols as Table 2.

Sheep 21, tests 1-5 over 1 year, 6, 7, 8 over 1 month.

Sheep 879, tests 1 and 2, 5 months interval, tests 2-4 over 1 month.

Sheep 9, tests 1 and 2,4 months interval, tests $2-4$ over 1 month.

Table 4. Distribution of different serological types of the Gram-negative coccus in two calves

Calf 1

$\%$ of isolations belonging $\%$ of isolations belonging

\begin{tabular}{|c|c|c|c|c|c|c|c|c|c|}
\hline \multirow{2}{*}{$\begin{array}{c}\text { Age } \\
\text { of calf } \\
\text { (weeks) }\end{array}$} & \multicolumn{4}{|c|}{ to coccus type } & \multicolumn{4}{|c|}{ to coccus type } & \multirow{2}{*}{$\begin{array}{l}\text { Total count } \\
\text { for each } \\
\text { calf } / \mathrm{ml} \text {. }\end{array}$} \\
\hline & 1 & 2 & 3 & 4 & 1 & 2 & 3 & 4 & \\
\hline 3 & . & . & . & . & . & . & . & . & $10^{10 *}$ \\
\hline \multirow[t]{2}{*}{4} & 0 & 100 & 0 & 0 & 100 & 0 & 0 & 0 & $10^{10}$ \\
\hline & - & ++ & & & - & \pm & & & \\
\hline \multirow[t]{2}{*}{6} & 0 & 100 & 0 & 0 & 96 & $\overline{4}$ & 0 & 0 & $10^{10}$ \\
\hline & - & + & & & - & - & & & \\
\hline \multirow[t]{2}{*}{8} & 5 & 16 & 79 & 0 & 100 & 0 & 0 & 0 & $10^{8}$ \\
\hline & - & + & & & + & \pm & & & \\
\hline \multirow[t]{2}{*}{10} & 0 & 23 & 62 & 15 & 50 & $\overline{0}$ & 50 & 0 & $10^{9}$ \\
\hline & - & + & & & - & - & & & \\
\hline \multirow[t]{2}{*}{12} & 0 & 0 & 80 & 20 & 0 & 0 & 100 & o & $10^{9}$ \\
\hline & - & - & & & \pm & - & & & \\
\hline \multirow[t]{2}{*}{14} & 8 & 0 & 0 & 92 & $\overline{9} 3$ & 0 & 7 & 0 & $10^{8}$ \\
\hline & - & - & & & + & \pm & & & \\
\hline
\end{tabular}

,,-+ Fluorescent antisera tests, symbols as Table 2.

* Not tested serologically.

from calves yielded three distinct serological types, with a few organisms belonging to what appeared to be a fourth type. A fifth type was isolated from sheep 879. The relationships between the types are shown in Table 5. There was some cross-reaction between the organisms and antisera types 1 and 2 which was discernible with the fluorescent antisera, and was apparent with slightly diluted (e.g. 1/80; titre with homologous organism $1 / 5,120$ ) antisera in 
tube agglutination tests. This cross-reaction was removed by absorbing the antisera with the appropriate organisms. The different serological types have been stable on sub-cultivation for about 2 years, with the exception of type 4 which rapidly lost the antigen which reacts with type 2 antiserum. No crossagglutination reaction of antisera types 1 or 2 with strains of Neisseria catarrhalis, Veillonella gazogenes or Sarcina bakeri (a rumen Gram-negative coccus) occurred. The dilute $\mathrm{NaOH}$ extract of any one serological type reacted in precipitin tests with all the antisera, suggesting the presence of a common group antigen. No group-reacting substance was extracted by the acid or formamide methods usually used for streptococci.

\section{Table 5. The relationships of the serological types of Gram-negative} cocci as shown by agglutination tests

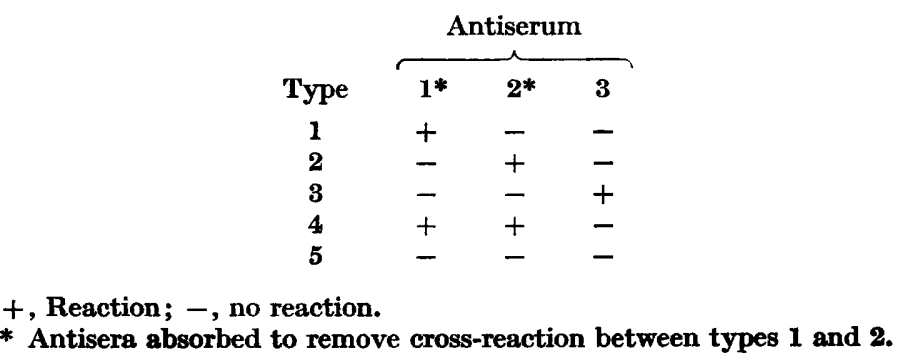

Serological type 2 is the most common in the calf rumen, nearly all the isolations from the calves mentioned in Table 2 which were tested belonged to this type, with a few type 1 . Types 3 and 4 only appeared in the later tests on calves 1 and 2. However, the results of detailed examination of the calf rumen isolations given in Table 4 show that the serological types fluctuate quite markedly and it is possible that more extended observations on the calves mentioned in Table 2 would have shown a wider variety of types. The discrepancy between the observations with fluorescent antisera and tests on isolated cocci from calf 2 at 4 and 6 weeks old cannot be explained. Samples from other calves from which no isolations were made have been tested with the fluorescent antisera and organisms of types 1 and 2 have been found, together with some morphologically similar cocci not reacting with either antiserum. The calf serological types 1 and 2 were found in sheep as well as a new type (5).

\section{Attempted establishment of the organism in the rumen}

In an attempt to establish the organism in large numbers in the rumen of a sheep a suspension of cocci of serological type 2 , from a $48 \mathrm{hr}$. CJA culture, containing approximately $5 \times 10^{9}$ cocci, was injected through the cannula into the rumen of a sheep which showed only type 5 cocci. Viable counts of the cocci, serological tests on the isolated organisms, and tests of the rumen contents with type 2 fluorescent antiserum were made at intervals up to 7 days after the inoculation, but the added organisms did not grow. The sheep had 
been selected because it had $10^{4}$ or less type 5 cocci $/ \mathrm{ml}$. rumen contents, but on the day of inoculation this had gone up to $10^{6} / \mathrm{ml}$.

Cultures were also made from throat swabs of sheep 21 at intervals. However, few large Gram-negative cocci were seen in films made from the swabs, and although some grew in culture, the main organisms cultured were Veillonella gazogenes.

\section{DISCUSSION}

From a comparison of their morphological and biochemical properties there is no doubt that the organism described here is that described by Elsden et al. (1956) under the name LC, and who discussed the taxonomic position of the organism and its pathway of lactate fermentation. However, these authors although they obtained the organism from a number of sheep did so by an indirect method, using medium $A$ as an enrichment culture and had no knowledge of the numbers present in sheep, or the distribution of the organism in other animals. Information about this, and about some of the serological properties of the organism, together with other properties not discussed by Elsden et al. (1956), are given in this paper. The results obtained would seem to show that while the organism is one of the major ones present in the rumen of the young animal it is not present in sufficient numbers in the adult animal to be of great significance. Even the ingestion of a diet containing large amounts of lactic acid, such as the silage fed to the heifers (Table 2), did not lead to the growth of an appreciable number of the cocci. (Cultural examination of the silage itself showed no large cocci.) The reason for the diminution in numbers as the animal grows older is being investigated. Microscopic observations on some calves of about 3 weeks old suggested a correlation between numbers of Gram-negative cocci and numbers of large Gram-positive rods (presumably lactobacilli) but no cultural work to confirm this has been done. The sheep isolations, and the failure of the attempt to establish the organism in the rumen of a sheep, suggest that there is some mechanism which limits the total numbers in the adult animal to less than $c .10^{6} \mathrm{cocci} / \mathrm{ml}$. The latter experiment and the need, in general, for a large inoculum to start cultures growing make it somewhat difficult to understand how the organism becomes established in the rumen, and the variation in numbers suggests that it is not well adapted to conditions there. The possibility that the organism's natural habitat is the throat and that the organisms in the rumen come from there led to the attempted isolation of the coccus from throat swabs. The results obtained so far do not support this possibility, as the small number of organisms isolated could quite easily have come from particles of food lodged in the throat after regurgitation from the rumen. The fluctuations in serological types in the calf rumen (Table 4) are quite wide, but similar results have been found for changes in serological types of Escherichia coli in the human intestinal tract (Sears \& Brownlee, 1952).

In animals from which the organism was not isolated large Gram-negative cocci (not reacting with the fluorescent antisera) were often seen, and it would appear that the coccus described here is only one of a number of morphologi- 
cally similar organisms in the rumen. A large Gram-negative tetracoccus (Sarcina bakeri), which also occurs in diplococcal form, was isolated some years ago from the sheep rumen (Mann, Masson \& Oxford, 1954), but this has no relationship to the coccus described here, and further tests where we have failed to isolate $\boldsymbol{S}$. bakeri, or detect it serologically, suggest that this, as well, is only one of a number of Gram-negative diplo- and tetra-cocci present in the rumen.

The large Gram-negative coccus was nearly always accompanied, in the lower dilutions at least, in rumen samples incubated in a lactate-containing medium, by Veillonella gazogenes, and the numbers in which these occurred supported the observations of Johns (1951) and Gutierrez (1953) that V. gazogenes is generally present in numbers of about $10^{4} / \mathrm{ml}$. sheep rumen contents. In the calves and heifers they were usually present in about the same number, although they were once found at $10^{8} / \mathrm{ml}$. in a heifer. However, direct observation of sheep or cattle rumen contents shows the presence of very large numbers of small Gram-negative organisms which might be $V$.gazogenes, and this does not accord with the comparatively small numbers isolated.

The authors wish to thank Miss M. Garvock and Miss S. Bell for technical assistance, and a number of colleagues for providing rumen samples.

\section{REFERENCES}

Browning, C. H. \& Mackie, T. J. (1949). Textbook of Bacteriology. 11th ed. Oxford University Press.

ELsDEN, S. R. (1946). The application of the silica gel partition chromatogram to the estimation of volatile fatty acids. Biochem. J. 40, 252.

ELSDEN, S. R. \& LEwIs, D. (1953). The production of fatty acids by a Gram-negative coccus. Biochem. J. 55, 183.

Elsden, S. R., Volcani, B. E., Gilchrist, F. M. C. \& Lewis, D. (1956). Properties of a fatty acid forming organism isolated from the rumen of sheep. J. Bact. 72, 681.

Friedemann, T. E. (1938). The identification and quantitative determination of volatile alcohols and acids. J. biol. Chem. 123, 161 .

GutIERREz, J. (1953). Numbers and characteristics of lactate utilising organisms in the rumen of cattle. J. Bact. 66, 128.

Hobson, P. N. \& MaNn, S. O. (1957). Some studies on the identification of rumen bacteria with fluorescent antibodies. J. gen. Microbiol. 16, 463.

Hobson, P. N., Mann, S. O. \& Oxford, A. E. (1958). The properties of a Gramnegative coccus from the rumen of the calf. $J$. gen. Microbiol. 18, i.

JoHns, A. T. (1951). Isolation of a bacterium, producing propionic acid, from the rumen of sheep. J. gen. Microbiol. 5, 317.

Mann, S. O., Masson, F. M. \& OxFord, A. E. (1954). Facultative anaerobic bacteria from the sheep's rumen. J. gen. Microbiol. 10, 142.

MARKham, R. (1942). A steam distillation apparatus suitable for micro-kjeldahl analysis. Biochem. J. 36, 790.

Moyle, V., Baldwin, E. \& Scarisbrick, R. (1948). Separation and estimation of saturated $\mathrm{C}_{2}-\mathrm{C}_{8}$ fatty acids on buffered partition columns. Biochem. J. 43, 308.

Rogosa, M., Wiseman, R. F., Joyce, A. M., Disraely, M. N. \& Beaman, A. J. (1953). Species differentiation of oral lactobacilli from man including descriptions of Lactobacillus salivarius nov. spec. and Lactobacillus cellobiosus nov. spec. J. Bact. 65, 681. 
Sears, H. J. \& Browntee, I. (1952). Further observations on the persistence of individual strains of Escherichia coli in the intestinal tract of man. J. Bact. 63, 47.

\section{EXPLANATION OF PLATE}

Figs. 1-4 are phase contrast, $\times 704$, of wet mounts taken directly from cultures.

Fig. 1. Normal growth of the large Gram-negative coccus.

Fig. 2. Older culture showing larger forms, and also chain formation normally seen mainly in young cultures.

Fig. 3. Older culture showing many swollen forms.

Fig. 4. Final stages of a culture showing swollen and disintegrating cells.

Fig. 5. Coccus type 2 , in the rumen contents of a 2 month old calf receiving a solid diet, reacting with fluorescent antisera. Background fluorescence blue-white. Ultra-violet, $\times 680$.

Fig. 6. Same field as fig. 5 . White light phase contrast $\times 680$.

(Received 16 May 1958) 
Journal of General Microbiology, Vol. 19, No. 3

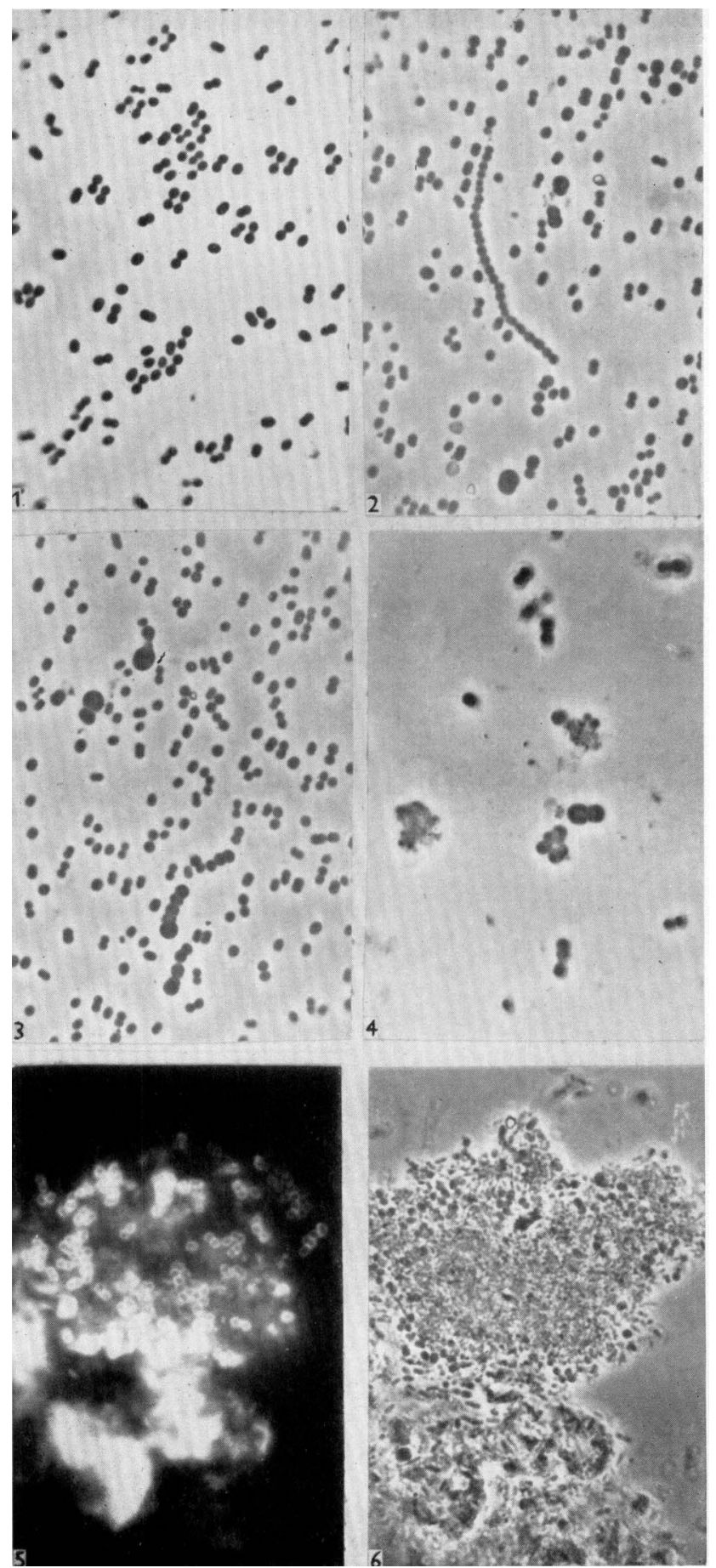

P. N. Hobson, S. O. Mann \& A. E. Oxford-A rumen Gram-negative coccus. Plate 1 